\title{
CUIDADOS NUTRICIONAIS NO RECÉM-NASCIDO DE MUITO BAIXO PESO
}

\section{NUTRITIONAL CARE OF VERY LOW BIRTH WEIGHT PRETERM INFANTS}

\author{
Adriana Gonçalves de Oliveira* \\ Pollyanna Patriota Siqueira** \\ Luiz Carlos de Abreu
}

Oliveira AGde, Siqueira PP, Abreu, LCde. Cuidados Nutricionais no Recém-nascido de Muito Baixo Peso. Rev Bras Crescimento Desenvol Hum. 2008; 18(2): 148-154.

\begin{abstract}
Resumo: Introdução: a meta nutricional na abordagem do recém-nascido de muito baixo peso é alcançar crescimento pós-natal em uma taxa que se aproxime do crescimento e do ganho de peso intra-uterino de um feto normal de mesma idade gestacional, sem produzir deficiências nutricionais, efeitos metabólicos indesejáveis ou toxicidades decorrentes de uma exagerada oferta nutricional. Objetivo: descrever o estado da arte da nutrição em recém-nascido prematuro de muito baixo peso. Método: Foram consultadas as bases de dados do Medline e SciELO. Utilizou-se como estratégia de busca no MEDLINE nutrition AND birth OR prematurity OR very low birth weigth infants e no SciELO os termos nutrition in newborn AND prematurity AND very low birth weigth infants AND nutritional status em todos os campos. Resultados: os artigos foram classificados quanto ao tipo: revisão, artigo original, relato de casos, opinião e editorial $(\mathrm{N}=16) \mathrm{e}$ compuseram a premissa do trabalho. Foram considerados estudos de natureza qualitativa e quantitativa. Os artigos listados de 1 a 4 foram utilizados para definir recém-nascido de muito baixo peso, pequeno para a idade gestacional e caracterização fisiológica da prematuridade. Conclusão: o início precoce de alimentação, tanto enteral como parenteral e a avaliação nutricional são imprescindíveis para garantir o crescimento e desenvolvimento neuropsicomotor adequados e evitar sequielas futuras nos recém-nascidos de muito baixo peso ao nascer.
\end{abstract}

Palavras-chave: Nutrição; muito baixo peso; prematuro; avaliação nutricional.

\section{INTRODUÇÃO}

Os recém-nascidos de muito baixo peso ao nascer (RNMBP) são aqueles que apresentam peso ao nascer $<1.500 \mathrm{~g} .{ }^{1}$ Quanto à gestação, são prematuros (PT) aqueles com nascimento menor que 37 semanas. ${ }^{1}$ A definição mais aceita de pequenos para a idade gestacional é a que considera Pequeno para a Idade Gestacional (PIG) como os nascidos vivos que apresentam valor inferior ao percentil 10 de peso ao nascer segundo idade gestacional. ${ }^{2}$
O peso ao nascer é o melhor preditor do padrão de saúde imediato e futuro do recém-nascido ( $\mathrm{RN}$ ), sendo que naqueles $\mathrm{RN}$ com peso ao nascer menor do que $1.500 \mathrm{~g}$ e idade gestacional inferior a 32 semanas, existem relações diretas de fatores de risco de instalação da hemorragia peri-intraventricular, à sobrevida e ao crescimento e desenvolvimento, sendo o muito baixo peso considerado relevante na taxa de mortalidade infantil. $^{3}$

O nascimento antes do final do $3^{\circ}$ trimestre predispõe o $\mathrm{RN}$ ao risco nutricional, em ra-

\footnotetext{
* Médica Neonatologista. Responsável pelo setor de Cuidados Intermediários do Hospital Maternidade Leonor Mendes de Barros, São Paulo, SP. Contato: Hospital Maternidade Leonor Mendes de Barros da Secretaria de Estado da Saúde, Estado de São Paulo. Neonatologista do Hospital Municipal de Diadema, SP. E-mail: agdeoliveira@ gmail.com.

** Nutricionista. Mestre em Saúde Materno-infantil. Docente da Universidade Federal de Alagoas - UFAL.

**** Pós-doutor em Saúde Pública. Docente do programa de Stricto sensu em Ciências da Saúde da Faculdade de Medicina do ABC, Santo André, SP.
} 
zão dos baixos níveis de nutrientes, crescimento rápido, imaturidade do trato gastrointestinal. ${ }^{4}$

A meta nutricional na abordagem do recém-nascido de muito baixo peso é alcançar crescimento pós-natal em uma taxa que se aproxime do crescimento e do ganho de peso intra-uterino de um feto normal de mesma idade gestacional, sem produzir deficiências nutricionais, efeitos metabólicos indesejáveis ou toxicidades decorrentes de uma exagerada oferta nutricional. ${ }^{4}$

Entretanto, o estado nutricional de um RN ao nascimento varia de acordo com as condições de vida intra-uterina as quais esteve submetida. A adequação nutricional do feto pode influenciar de forma significante a morbidade e mortalidade do RN. Nascer prematuramente coloca o RN numa condição de grande risco nutricional. A alimentação representa contínuo desafio para os responsáveis pela nutrição do neonato, principalmente daqueles prematuros e de muito baixo peso ao nascer.

Quando as conseqüências nutricionais e metabólicas decorrentes das deficiências são significativas, levam a alterações em curto e longo prazo, tais como repercussão no desenvolvimento cerebral, déficit de aprendizado e déficit de memória. Assim, o objetivo é descrever o estado da arte da nutrição em recém-nascido prematuro de muito baixo peso.

\section{MÉTODO}

Foram consultadas as bases de dados: MEDLINE/PUBMED (Literatura Internacional em Ciências da Saúde) e SciELO (Scienfic Ele- tronic Library). Publicações indexadas no Medline, mesmo que em revistas nacionais, foram consideradas internacionais. As consultas incluíram somente artigos registrados entre 1/1/2001 a 30/08/2008.

As seguintes estratégias de busca foram utilizadas:

MEDLINE/PUBMED: utilizaram-se os termos de busca nutrition AND birth OR prematurity OR very low birth weigth infants para encontrar os trabalhos de que contivessem o termo em qualquer campo. Foram usados como limites de busca: subset Medline e referências nos idiomas inglês e português.

SciELO: em busca avançada, pesquisouse ano a ano (2001 a 2008), utilizando o termo nutrition in newborn AND prematurity AND very low birth weigth infants AND nutritional status em todos os campos. A seleção dos artigos foi realizada manualmente e excluídos os que mencionavam nutrition sem a ligação com newborn. Os artigos duplicados foram excluídos nas bases de dados, prevalecendo aquela de maior abrangência e na seguinte ordem: MEDLINE/ PUBMED e SciELO. Os periódicos que utilizam a metodologia SciELO foram considerados como desta base.

Os artigos foram classificados quanto ao tipo: revisão, artigo original, relato de casos, opinião e editorial $(\mathrm{N}=16)$ e compuseram a premissa do trabalho. Foram considerados estudos de natureza qualitativa e quantitativa. Ainda, os artigos listados de 1 a 4 foram utilizados para definir termos: recém-nascido de muito baixo peso ${ }^{1}$, pequeno para a idade gestacional ${ }^{2}$ e caracteriza-

Tabela 1: Distribuição dos manuscritos nas bases de dados MEDLINE/PUBMED e SciELO no período de 2001 a 2008.

\begin{tabular}{llll}
\hline TIPO DE MANUSCRITO & \multicolumn{3}{c}{ BASE DE DADOS } \\
\hline \\
Experimentais & SciELO & MEDLINE/PUBMED & Total \\
Revisão & 01 & 13 & 14 \\
Opinião & 01 & - & 01 \\
Total & - & 01 & 01 \\
\hline
\end{tabular}

Scielo: Scientific Electronic Library Online

Medline: Medical Literature Analysis and Retrieval System Online 
Tabela 2: Síntese dos manuscritos que versaram sobre nutrição do recém-nascido de muito baixo peso no período de 2006 a 2008, nas bases de dados do SciELO e MEDLINE/PUBMED.

\begin{tabular}{ll}
\hline Brock, Falcão e Leoni & \\
Benevenuto et al. ${ }^{10}$ & $\begin{array}{l}\text { O uso de um instrumento validado para o período neonatal é imprescindível ao } \\
\text { acompanhamento dos RNs. }\end{array}$ \\
& $\begin{array}{l}\text { A prevalência de aleitamento exclusivo e misto em RNPT após alta hospitalar foi mais } \\
\text { evidenciado que noutros estudos similares, destacando a necessidade de prover condições } \\
\text { para seqüência do aleitamento para esta população o máximo possível. }\end{array}$ \\
Cardoso e Falcão ${ }^{20}$ & O IMC é o melhor índice para avaliação antropométrica dos RNMBP. \\
Elizabeth et al. ${ }^{11}$ & Prematuridade isolada ou associada à infecção requer suplementação de nutrientes. \\
Doege e Bauer ${ }^{17}$ & $\begin{array}{l}\text { A nutrição precoce por dieta enteral previne atrofia e estimula a maturação do trato } \\
\text { gastrintestinal, além de previnir a enterocolite necrosante. }\end{array}$ \\
Brock e Falcão & O peso de nascimento e prematuridade está diretamente relacionado com a presença de \\
& síndrome metabólica nos adultos.
\end{tabular}

RNPTMBP= recém-nascido pré-termo de muito baixo peso

$\mathrm{IMC}=$ índice de massa corpórea

$\mathrm{RN}=$ recém-nascido

Tabela 3: Síntese dos manuscritos que versaram sobre nutrição do recém-nascido de muito baixo peso no período de 2001 a 2005, nas bases do SciELO e MEDLINE/PUBMED.

\begin{tabular}{|c|c|}
\hline Camelo $^{5}$ & $\begin{array}{l}\text { Baixo peso e desnutrição intra-útero caminham juntos, aumentando a morbidade e } \\
\text { mortalidade e levando a um desenvolvimento cognitivo pobre. }\end{array}$ \\
\hline Gianini et al. ${ }^{9}$ & Melhora na evolução clínica é diretamente proporcional há melhora da oferta nutricional. \\
\hline Cooke et al. ${ }^{14}$ & $\begin{array}{l}\text { A falta de ingestão calórica e protéica adequadas corrobora para o retardo de crescimento } \\
\text { pós-natal. }\end{array}$ \\
\hline Hust et al. ${ }^{12}$ & $\begin{array}{l}\text { Minimização do risco de desnutrição em RNPTMBP pode ser verificada com adequada } \\
\text { avaliação nutricional. }\end{array}$ \\
\hline Lubetzky et al. ${ }^{16}$ & $\begin{array}{l}\text { O leite materno é menos dispendioso do ponto de vista energético do que a alimentação } \\
\text { com fórmula pelo prematuro. }\end{array}$ \\
\hline Cooke e Foulder-Hughes ${ }^{18}$ & $\begin{array}{l}\text { Os déficits de crescimento e desenvolvimento do prematuro são diretamente relacionados } \\
\text { aos aspectos nutricionais. }\end{array}$ \\
\hline Ziegler et al. ${ }^{15}$ & $\begin{array}{l}\text { Uso de nutrição parenteral e enteral agressiva como modelo de redução das alterações do } \\
\text { crescimento e desenvolvimento. }\end{array}$ \\
\hline Embleton et al. ${ }^{13}$ & A falta de ingestão calórica e protéica constitui um problema universal. \\
\hline
\end{tabular}

RNPT = recém-nascido pré-termo

UTI= Unidade de Terapia Intensiva

RNPTMBP= recém-nascido pré-termo de muito baixo peso

ção fisiológica da prematuridade. ${ }^{3 \mathrm{e} 4}$

O conceito de prematuridade ${ }^{1,3,4}$ inclui todo recém-nascido (RN) vivo com menos de 37 semanas completas de gestação ( $<259$ dias) contadas a partir do primeiro dia do último período menstrual. Os recém-nascidos pré-termo com peso inferior a $1500 \mathrm{~g}$ são denominados pré-termo de muito baixo peso. ${ }^{1}$

\section{RESULTADOS}

Foram encontrados no MEDLINE/PUB- 
MED 28 artigos versando sobre os descritores nutrição OR nutricion. Com a adição do termo recém-nascido de muito baixo peso (nutrição OR nutrition AND very low birth weigth infants) reduziu-se o número de manuscritos encontrados. Após exclusão dos artigos não pertinentes ao estudo, 18 manuscritos permaneceram e foram considerados como de publicações sobre nutrição de recém-nascidos de muito baixo peso.

Destes, 16 artigos são originais, um de revisão e um de opinião (tabela 1), sendo que 14 $(87,5 \%)$ foram publicados na base de dados do MEDLINE/PUBMED (13 experimentais e um de opinião), sendo que dois $(12,5 \%)$ foram publicados na base de dados da Scielo (um experimental e um de revisão).

$\mathrm{Na}$ base de dados da SciELO foram encontrados 18 artigos, dos quais 12 foram excluídos por não serem sobre nutrição no RNPTM$\mathrm{BP}$ e quatro por estarem duplicados com a base MEDLINE/PUBMED.

\section{DISCUSSÃO}

O Comitê de Nutrição das Nações Unidas e do Banco Mundial estimam que nasçam anualmente cerca de 30 milhões de crianças com retardo de crescimento intrauterino (RCIU) em países em desenvolvimento. Baixo peso e desnutrição intra-útero caminham juntos, aumentando a morbidade e mortalidade e levando a um desenvolvimento cognitivo pobre. Os recém-nascidos de muito baixo peso (RNMBP) acabam sendo submetidos a processos prolongados de desnutrição extra-uterina, que agravam a sua situação clínica, criando problemas potencias para o seu futuro, tanto nos processos de crescimento e desenvolvimento quanto na possibilidade de surgimento de doenças metabólicas e degenerativas na infância, adolescência e idade adulta. ${ }^{5}$

O período desde a concepção até o nascimento é caracterizado por impressionante crescimento e desenvolvimento dos tecidos. $\mathrm{O}$ crescimento do feto está intimamente vinculado à oferta de oxigênio e nutrientes e é influenciado por fatores genéticos, importantes no início da gestação e ambiente materno, importante no final da gestação. Os fatores maternos que mais influenciam o crescimento fetal são: paridade, nível sócio-econômico, raça, altura, fumo, estado nutricional, unidade útero-placentária e hormônios. ${ }^{6}$

O termo restrição do crescimento intrauterino (RCIU) indica que o RN pequeno para idade gestacional poderia ter sido maior caso não houvesse fatores maternos e ou fetais que prejudicassem o seu crescimento. Os recém-nascidos pequenos para idade gestacional (PIG) apresentam maior mortalidade e dificuldade de adaptação neonatal em curto e longo prazo, mostrando que o déficit de crescimento é uma condição patológica ou de doença, sendo adequado manter certo grau de vigilância. ${ }^{6}$

Anchieta et al. ${ }^{7}$ avaliaram o crescimento de recém-nascidos pré-termo nas primeiras 12 semanas de vida, que receberam dieta enteral já na $1^{\circ}$ semana e atingiram as necessidades calóricas no final da $2^{\circ}$ semana de vida. Todos os recém-nascidos apresentaram perda de peso e recuperação após, mas isto dependeu do aporte hídrico e calórico oferecido indicando que, se não for ofertado um adequado suporte nutricional, a recuperação do crescimento será mais lenta.

Estudo recente ${ }^{9}$ demonstrou que prevalência de desnutrição ao atingir o termo foi $63,5 \%$. Neste mesmo estudo, os PIG ao nascimento foram 12 vezes mais desnutridos. Outrossim, há uma melhora na avaliação nutricional quando os RN apresentam melhor evolução clínica, pois conseguem receber alimentação precocemente (parenteral e/ou enteral) e leite materno aditivado ${ }^{9}$. Entretanto, Benevenuto et al. ${ }^{10}$ estudaram RNMBP que receberam leite humano durante o período de internação e relatam que a média de aleitamento materno exclusivo após alta nesta população foi menor em relação ao aleitamento misto até o final do $6^{\circ}$ mês de vida. ${ }^{10}$

Os RNT e os RNPT com baixo peso nascem com menor reserva de nutrientes, sendo esta caracterização mais evidenciada nos RNPT. Ainda, estas reservas podem ser diminuídas em casos de infecção e nutrição inadequada, o que leva a necessidades de suplementação de nutrientes nesta população. ${ }^{11}$

Hust et al. ${ }^{12}$ relatam que a ingestão inadequada de nutrientes e/ou interrupção da ali- 
mentação durante a hospitalização são fatores diretamente relacionados com a desnutrição protéico-calórica, a qual leva a um aumento da morbidade como alterações no crescimento e desenvolvimento neuropsicomotor, e a um aumento da mortalidade. Destaca-se que no RNPT há uma alta taxa metabólica e requerimento energético por $\mathrm{Kg}$ de peso corporal e alto turnover protéico, com alto catabolismo em situações críticas e de suporte nutricional deficiente. Sugerem que uma avaliação nutricional inicial corrobora para a redução dos danos neurológicos ocasionados por esta condição de alta taxa metabólica e baixa oferta de nutrientes. ${ }^{12}$

Para Embleton et al..$^{13}$ o retardo pós-natal é um problema universal, não importando onde o $\mathrm{RN}$ esteja. Isto é resultado de uma falta de ingestão calórica e protéica, a qual é interrompida com freqüência e não é reposta na necessidade do RN sob condições críticas do crescimento e desenvolvimento. Desta maneira, a dieta parenteral introduzida precocemente, bem como a dieta enteral com um volume adequado reduz este déficit. Destacam ainda que esta adequação nutricional deverá ser realizada com os RN estáveis clinicamente e tolerantes à dieta prescrita. ${ }^{13}$ É necessário um suporte nutricional especializado para estes $\mathrm{RN}$ de alto risco, para prevenir o retardo de crescimento no primeiro ano de vida. ${ }^{14}$

A desnutrição no período inicial da vida pode ter efeitos permanentes no desenvolvimento do sistema nervoso central, na cognição e crescimento somático. Em algumas idades, o requerimento protéico é considerado mais certo do que o energético, o qual ainda é incerto. Nos RNMBP ainda há poucos dados em relação a isto. Considerouse, neste estudo, um gasto de 15 a $20 \mathrm{Kcal} / \mathrm{Kg} / \mathrm{dia}$ nos RN com peso menor que $1200 \mathrm{~g}$, embora parecesse haver evidências que o gasto energético no repouso destes RN é baixo, principalmente na $1^{\circ}$ sem de vida. ${ }^{15}$

Ziegler et al. ${ }^{15}$ propuseram o acesso mais agressivo à nutrição do RNMBP, o qual propicia a redução da privação nutricional e seu efeito sobre o crescimento e desenvolvimento do RNMBP. Na nutrição enteral, a prática corrente é a recusa em alimentar por período prolongado para prevenir a enterocolite necrosante, assim como em RN com instabilidade cardiovascular. Freqüentemente, os RN têm resíduo gástrico, induzindo a um diagnóstico de intolerância a alimentação. Como prática agressiva, o objetivo da alimentação nos primeiros dias de vida é estimular a liberação dos hormônios intestinais, assim como estimular sua maturação e motilidade. $\mathrm{O}$ alimento preferido é o leite materno e deve ser iniciado no dia do nascimento. Resíduo gástrico não deve ser seguido para interferir com a alimentação, assim como RN com instabilidade cardiovascular não é indicação da supressão da nutrição enteral. Dever-se-á iniciar com 1 a $2 \mathrm{ml} / \mathrm{vez}$ a cada 6 horas ou menos, preferencialmente a cada 3horas. Aumentar volume em $20 \mathrm{ml} / \mathrm{Kg} / \mathrm{dia}^{15}$.

Para os RNMBP, o leite deve ser aditivado, iniciando antes mesmo de atingir um volume máximo $(100 \mathrm{ml} / \mathrm{Kg} / \mathrm{dia})$, dando em torno de 3,1 a 3,25g/100 Kcal de proteína. O leite materno contém em torno de $1,0 \mathrm{~g} / 100 \mathrm{Kcal}$ e as fórmulas contém entre 2,7 e $3,0 \mathrm{~g} / 100 \mathrm{Kcal} .{ }^{15}$ Por outro lado, o requerimento energético depende de cada $\mathrm{RN}$, do seu ganho de peso, da sua tolerância do volume e deve ser ajustado diariamente. ${ }^{15} \mathrm{O}$ RNPT tem um menor gasto energético quando alimentados com leite materno, comparando com aqueles alimentados com fórmula. ${ }^{16}$

A introdução precoce da dieta enteral previne atrofia e estimula a maturação intestinal, prevenindo complicações como enterocolite necrosante $(\mathrm{ECN})$. O volume mais elevado foi bem tolerado pelos prematuros, promovendo um crescimento adequado quando comparado com o crescimento intra-uterino. Os nutrientes do leite materno podem ser insuficientes para o crescimento do PT, sendo necessária a suplementação com proteína e fósforo, melhorando o ganho de peso, a resposta metabólica e a mineralização óssea. ${ }^{17}$

Em estudo longitudinal ${ }^{18}$ de RNPT avaliados aos 7 anos de idade, observou-se alterações típicas do desenvolvimento e crescimento da criança, tais como menor desempenho motor, menor índice no teste de inteligência, menor perímetro cefálico (PC) e índice de massa corpórea (IMC), além de déficit de crescimento e desenvolvimento cognitivo em longo prazo e lentidão nas atividades. Estas condições clínicas foram 
relacionadas diretamente ao fatores nutricionais durante internação e no período pós-natal dos RNMBP e PIG. ${ }^{18}$

Noutro estudo, Clark et al. ${ }^{19}$ destacam que prematuridade, baixo peso, sexo, necessidade de suporte ventilatório, presença de ECN e uso de esteróides, estão relacionados com déficit de crescimento no período pós-natal e dificuldade na nutrição. Isto leva a uma dificuldade neurosensorial e um pobre acompanhamento escolar, principalmente aqueles que têm o PC com 8 meses abaixo do esperado.

Cardoso e Falcão ${ }^{20}$ correlacionaram os valores das medidas antropométricas RNPTMBP e demonstraram que o IMC (peso/comp ${ }^{2}$ ) é o parâmetro mais fidedigno em demonstrar o crescimento real destas populações.

De maneira continuada buscam-se instrumentos que possam mensurar estes déficts propiciados no pré-natal e no pós-natal. A avaliação do crescimento fetal tem sido representada pelas curvas de crescimento intra-uterino. Ademais, os métodos de avaliação nutricional pós-natal, tais como parâmetros bioquímicos, parâmetros antropométricos, avaliação da composição corpórea por bioimpedância eletromagnética (BIA) e as relações antropométricas, deverão ser utilizadas para a avaliação e determinação do IMC no período neonatal e em diferentes idades gestacionais. Estes instrumentos tendem a relatar que o peso de nascimento e não só a prematuridade está inversamente relacionado à mortalidade por doença cardiovascular e à síndrome de resistência a insulina que engloba elevação da pressão arterial, intolerância à glicose e aumento dos níveis séricos de triglicérides (síndrome metabólica). ${ }^{7,8}$

Assim, o início precoce de alimentação, tanto enteral como parenteral, é fundamental para não alterar o estado nutricional, minimizar perdas e estimular o desenvolvimento do trato gastrointestinal, bem como a avaliação nutricional antropométrica periódica é fundamental para acompanhar o crescimento e desenvolvimento de acordo com o canal de crescimento e realizar intervenção bioquímica precoce quando necessário e o seguimento pós-alta se faz necessário para acompanhar o crescimento e desenvolvimento sem prejuízo nutricional.

Nos RNPTMBP, a nutrição deve receber cuidado especial para garantir o crescimento e o desenvolvimento neuropsicomotor adequados e evitar seqüelas futuras. A avaliação nutricional realizada através das medidas antropométricas e, mais recentemente, através do índice de massa corpórea deve ser contínua. A introdução de nutrição parenteral e/ou enteral precoce deve ser considerada uma rotina, com a finalidade de garantir um ganho de peso adequado e uma recuperação mais rápida durante a hospitalização deste recém-nascido.

\begin{abstract}
Introduction: the nutritional aim in the approach to the newborn with very low weight is to achieve post-natal growth at a rate that is similar to the intra-uterine growth and weight gain of a normal fetus of the same gestational age, without producing nutritional deficiencies, undesirable metabolic effects or toxicities resulting from excessive nutritional supply. Objective: to describe state-of-the-art nutrition in preterm infants with very low weight. Method: We consulted the databases of Medline and SciELO. The search strategy used in MEDLINE were the keywords nutrition AND birth OR prematurity OR very low birth weight infants, and in SciELO, the terms nutrition in newborn AND prematurity AND very low birth weight infants AND nutritional status in all fields. Results: the articles were classified according to type: review, original article, case report, opinion and editorial $(\mathrm{N}=16)$ and formed the premise of the study. We considered studies of qualitative and quantitative nature. The articles listed from 1 to 4 were used to define newborns with very low weight, small for gestational age and physiological characterization of prematurity. Conclusion: the early onset of nutrition, both oral and parenteral, and nutrition assessment are essential to ensure appropriate neuropsychomotor growth and development and to avoid future sequels in newborns with very low birth weight.
\end{abstract}

Keywords: Nutrition; very low birth weight infants; preterm infants; nutrition assessment. 


\section{REFERÊNCIAS}

1. Almeida MF, Novaes HMD, Alencar GP, Rodrigues LC. Mortalidade neonatal no Município de São Paulo: influência do peso ao nascer e fatores sócio-demográficos e assistenciais. Rev Bras Epidemiol., 2002; 5:93107.

2. Lubchenco LO, Hansman C, Dressler M. et al. Intrauterine growth as estimated from live-born birth-weight data at 24 to 42 weeks of gestation. Pediatrics, 32:793-800, 1963.

3. Abreu, LC, Souza, AMB, Oliveira, AG et al. Incidência de hemorragia peri-intraventricular em recém-nascidos pré-termo e a relação com o peso ao nascer. Rev. bras. crescimento desenvolv. hum., 2007, vol.17(2): 24-30.

4. Anderson D. Nutritional assessment and therapeutic interventions for the preterm infant. Clin Perinatol., 2002;29:313-26.

5. Camelo JS. Recém-nascido de muito baixo peso e estado nutricional: certezas e incertezas. J Pediatr (Rio J). 2005;81:5-6.

6. Brock RS, Falcão MC. Avaliação nutricional do recém-nascido: limitações dos métodos atuais e novas perspectivas. Rev Paul Pediatr 2008; 26(1):70-76.

7. Anchieta LM, Xavier CC, Colosimo EA. Crescimento de recém-nascidos pré-termo nas primeiras 12 semanas de vida. J Pediatr (Rio J). 2004;80(4);267-76.

8. Brock RS, Falcão MC, Leoni C. Body mass índex values for newborns according to gestational age. Nutr Hosp. 2008;23(5):487-492.

9. Gianini NM, Vieira AA, Moreira ME. Avaliação dos fatores associados ao estado nutricional na idade corrigida de termo em recém-nascidos de muito baixo peso. J Pediatr (Rio J). 2005;81:34-40.

10. Benevenuto MM, Thomson Z, Vannuchi MT, Matsuo T. Feeding patterns of brazilian preterm infants during the first 6 months of life, Londrina, Paraná, Brazil. J Hum Lact. 2007; 23(3), 269-74.
11. Elizabeth KE, Krishnan V, Zachariah P. Auxologic, Biochemical and Clinical (ABC) Profile of Low Birth Weight Babies. A 2-year Prospective Study. J Trop Pediatr. 2007; 53(6):374-82.

12. Hulst J, Joosten K, Zimmermann L et al.. Malnutrition in critically ill children: from admission to 6 months after discharge. Clin Nutr. 2004; 23(2); 223-232.

13. Embleton NE, Pang N, Cooke RJ. Postnatal Malnutrition and Growth Retardation: An Inevitable Consequence of Current Recommendations in Preterm Infants? . Pediatrics 2001;107:270-273.

14. Cooke RJ, Ainsworth SB, Fenton AC. Postnatal growth retardation: a universal problem in preterm infants. Arch Dis Child Fetal Neonatal Ed. 004;89:F428-30.

15. Ziegler EE, Thureen PJ, Carlson SJ. Aggressive nutrition of the very low birthweight infant. Clin Perinatol. 2002;29:225-244

16. Lubetzky R, Vaisman N, Mimouni FB, Dollberg $\mathrm{S}$. Energy expenditure in human milk- versus formula-fed preterm infants. J Pediatr. 2003 Dec;143(6):750-3.

17. Doege C, Bauer J. Effect of high volume intake of mother's milk with an individualized supplementation of minerals and protein on early growth of preterm infants $<28$ weeks of gestation. Clin Nutr. 2007 Oct;26(5):581-8.

18. Cooke RWI, Foulder-Hughes L. Growth impairment in the very preterm and cognitive and motor performance at 7 years. Arch Dis Child 2003;88:482-487.

19. Clark RH, Thomas P, Peabody J. Extrauterine growth restriction remains a serious problem in prematurely born neonates. Pediatrics. 2003; 111(5 Pt 1):986-90.

20. Cardoso LE, Falcão MC. Importância da avaliação nutricional de recém-nascidos prétermo por meio de relações antropométricas. Rev Paul Pediatr 2007;25:135-41. 